\title{
Influence of Threshold Stress on the Estimation of the Weibull Statistics
}

\author{
Chunsheng Lu \\ Institut für Struktur- und Funktionskeramik, Montanuniversität Leoben, A-8700 Leoben, Austria \\ State Key Laboratory of Nonlinear Mechanics, Institute of Mechanics, Academia Sinica, \\ Beijing 100080, People's Republic of China \\ Robert Danzer* \\ Institut für Struktur- und Funktionskeramik, Montanuniversität Leoben, A-8700 Leoben, Austria
}

Franz Dieter Fischer

Institut für Mechanik, Montanuniversität Leoben, A-8700 Leoben, Austria

The influence of threshold stress on the estimation of the Weibull statistics is discussed in terms of the Akaike information criterion. Numerical simulations show that, if sample data are limited in number and threshold stress is not too large, the two-parameter Weibull distribution is still a preferred choice. For example, the fit of strength data of glass and ceramics to the two- and three-parameter Weibull distributions is compared.

\section{Introduction}

CIMILAR to other brittle materials, measurement of the strength of a series of nominally identical ceramic specimens typically produces considerable scatter in the results. It is thus desirable to have some means of describing such a behavior quantitatively and further incorporating it into the assessment of reliability. ${ }^{1-3}$ Weibull first proposed such a means based mainly on the weakestlink hypothesis and a simple empirical function, which has been and is still widespreadly used in the description of fracture data. ${ }^{4-6}$ According to the Weibull statistics, the cumulative failure probability $F(\sigma)$ of a material subjected to a stress $\sigma$ is given by

$$
F(\sigma)=1-\exp \left[-\left(\frac{\sigma-\sigma_{\mathrm{th}}}{\sigma_{0}}\right)^{m}\right]
$$

where $\sigma_{0}$ is a normalized factor known as the characteristic strength, $\sigma_{\mathrm{th}}$ is the threshold stress (below which no failure will occur), and $m$ is the Weibull modulus. Here, the Weibull modulus $m$ is a measure of strength diversity, and is also called the shape factor. In most cases, for the sake of simplicity $\sigma_{\text {th }}$ is usually

A. Jagota-contributing editor

\footnotetext{
Manuscript No. 187235. Received January 2, 2002; approved April 2, 2002.

Supported by the Lise Meitner Program of Austrian Science Fund (FWF) under Project No. M587.

${ }^{*}$ Member, American Ceramic Society.
}

assumed to be 0 , and then the Weibull distribution can be reduced to a flexible two-parameter analytic form, such as

$$
F(\sigma)=1-\exp \left[-\left(\frac{\sigma}{\sigma_{0}}\right)^{m}\right]
$$

Up to now, there has been a lack of clear understanding of the effects of the stress threshold, and this can lead to an overestimation of the probability of failure when we adopt the fitted simple distribution in the prediction of failure of ceramic components. ${ }^{7}$ In this paper, we will suggest a simple quantitative procedure that can be applied to highlight the influence of the stress threshold on the estimation of the Weibull statistics.

\section{Akaike Information Criterion}

The best estimate of the unknown parameters in the Weibull distribution is the maximum likelihood method, which shows the smallest coefficient of variation while it is more cumbersome than the usually used linear-regression approach. ${ }^{8}$ Here, the loglikelihood for a given probability density function is defined as $\ln L=\sum_{i=1}^{N} \ln f\left(\sigma_{i}\right)$, where $\sigma_{i}$ is the strength of the $i$ th specimen, $N$ is the number of measurements, and $f(\sigma)$ is the probability density function, i.e., $f(\sigma)=\mathrm{d} F(\sigma) / \mathrm{d} \sigma$. Thus, for the threeparameter Weibull distribution, we have the following loglikelihood function:

$$
\ln L=\sum_{i=1}^{N} \ln \left\{\frac{m}{\sigma_{0}}\left(\frac{\sigma_{i}-\sigma_{\mathrm{th}}}{\sigma_{0}}\right)^{m-1} \exp \left[-\left(\frac{\sigma_{i}-\sigma_{\mathrm{th}}}{\sigma_{0}}\right)^{m}\right]\right\}
$$

The solution is found by maximizing the log-likelihood function and solving for $\left(\hat{m}, \hat{\sigma}_{0}, \hat{\sigma}_{\text {th }}\right)$ so that $\partial \ln L / \partial m=0, \partial \ln L / \partial \sigma_{0}=0$, and $\partial \ln L / \partial \sigma_{\text {th }}=0$.

The likelihood approach can be extended to making comparisons between models by the Akaike information criterion (AIC), ${ }^{9,10}$ which starts by linking the likelihood to a distance between the true and estimated distributions, and is defined as

$$
\mathrm{AIC}=-2(\ln \hat{L}-k)
$$

where $\ln \hat{L}$ is the maximum log-likelihood of a given model, $k$ is the number of parameters to be fitted in the model, and the additional factor 2 is a sop to historical precedents and could be omitted. 
Table I. $\Delta$ (AIC) Values Calculated for $m_{3 p}=10$, $\sigma_{03 \mathbf{p}}=1000 \mathrm{MPa}$

\begin{tabular}{cccccc}
\hline & \multicolumn{5}{c}{$\Delta($ AIC $)$} \\
\cline { 2 - 6 }$\sigma_{\text {th }} / \sigma_{03 \mathrm{p}}$ & $N=20$ & $N=40$ & $N=60$ & $N=80$ & $N=100$ \\
\hline 0.1 & -1.99 & -1.97 & -2.00 & -1.80 & -1.75 \\
0.2 & -1.95 & -1.89 & -2.00 & -1.65 & -1.57 \\
0.3 & -1.92 & -1.89 & -1.99 & -1.53 & -1.42 \\
0.4 & -1.90 & -1.90 & -1.98 & -1.43 & -1.30 \\
0.5 & -1.88 & -1.87 & 4.06 & 5.71 & 8.26 \\
\hline
\end{tabular}

Generally speaking, it is easier to fit a data set using a complex model with more parameters than a simple one with few parameters. The AIC represents a rough way of compensating for additional parameters and is a useful heuristic measure of the relative effectiveness of different models. It is, however, worth noting that the AIC values obtained here should be used with some caution since the amount of experimental data is usually not very large (e.g., standard requests $N \geq 30$ ). In typical cases, model differences which would be significant at around the 5\% confidence level correspond to differences in AIC values of around $1.5-2 .^{10-12}$ For example, in comparing the Weibull distribution with three parameters $(k=3)$ against the Weibull distribution with two parameters $(k=2)$, the former must demonstrate a significantly better fit to justify the additional parameter.

\section{Simulation Results and Discussion}

As is well known, many potential factors in real data could affect the Weibull distribution. ${ }^{13-18}$ But, this uncertainty can be easily resolved in numerical simulations. In the following, we imagine that the strength data of a fictitious material yield the three-parameter Weibull distribution. According to Eq. (1), the strength $\sigma$ is given by

$$
\sigma=\sigma_{\text {th }}+\sigma_{03 p}\{-\ln [1-F(\sigma)]\}^{1 / m_{3 \mathrm{p}}}
$$

Note here the subscript $3 p$ of parameters is used as a label to distinguish between Eqs. (1) and (2), and similar symbols will be used below.

Obviously the probability $F\left(\sigma_{i}\right)$ is a uniformly distributed random number between 0 and 1 , which can be generated by means of Monte Carlo simulations. ${ }^{19}$ In each trial a sample of size $N$ will be simulated, provided the three parameters in Eq. (5) and a random number seed are given. As a typical example, let us assume $m_{3 \mathrm{p}}=10, \sigma_{03 \mathrm{p}}=1000 \mathrm{MPa}$, and different threshold stresses $\sigma_{\text {th }}$ are realized through adjusting the ratio of $\sigma_{\mathrm{th}} / \sigma_{03 \mathrm{p}}$. Thus a series of data sets can be created, and each data set will then be applied to the fit of the two- and three-parameter Weibull distributions and further to the calculation of AIC values, as shown in Eq. (4).

Table I lists the difference of AIC values calculated by the twoand three-parameter Weibull distributions, that is, $\Delta$ (AIC) $=$ $(\mathrm{AIC})_{2 \mathrm{p}}-(\mathrm{AIC})_{3 \mathrm{p}}$. It is obvious that, if a sample is limited in number and the threshold stress is not too large, the difference of AIC values is not large enough for us to make a clear distinction between the two distributions (see Table I, where $\Delta$ (AIC) $<0$ in most cases). This implies that, in most applications, the flexible two-parameter Weibull distribution is still a good choice.

Next, strength data from three brittle materials with various levels of sample size, abraded Kimble R-6 soda-lime glass and

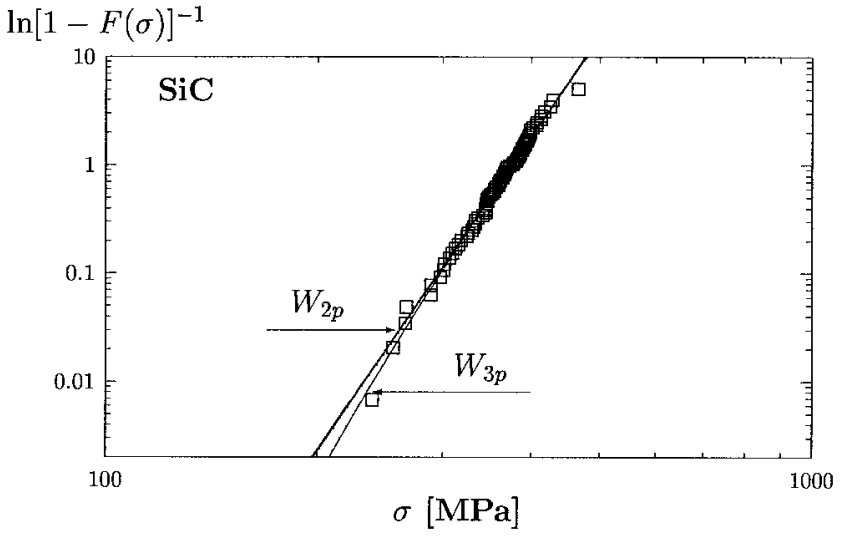

Fig. 1. Comparison of the two-parameter $\left(W_{2 \mathrm{p}}\right)$ with three-parameter $\left(W_{3 \mathrm{p}}\right)$ Weibull distributions for the fit of strength data of SiC.

two ceramics ( $\mathrm{SiC}$ and $\mathrm{Si}_{3} \mathrm{~N}_{4}$ ), are assessed. ${ }^{20,21}$ In general strength data are often arranged in ascending order, and each strength is assigned to a failure probability estimate of $F\left(\sigma_{i}\right)=$ $(i-0.5) / N$, where $i$ is the $i$ th strength and $N$ is the number of measurements. ${ }^{22}$ As shown in Table II, the three-parameter Weibull distribution cannot greatly improve the fit in all three cases although an additional parameter is introduced (where $\left.\Delta(\mathrm{AIC})=(\mathrm{AIC})_{2 \mathrm{p}}-(\mathrm{AIC})_{3 \mathrm{p}}<0\right)$. This can be seen from Fig. 1, where a $\log -\log$ plot of $\ln [1-F(\sigma)]^{-1}$ versus $\sigma$ for the SiC ceramic is illustrated. Here, it should be noted that different values of $m_{2 \mathrm{p}}$ and $\sigma_{02 \mathrm{p}}$ are obtained which are not comparable with the values of $m_{3 \mathrm{p}}$ and $\sigma_{03 \mathrm{p}}$.

Finally, it is worth pointing out that, as for various Weibull moduli $m_{3 p}$, the afore-mentioned phenomena have also been found by numerical simulations. But the larger the Weibull modulus, the smaller the region where there is not a clear distinction between the two- and three-parameter Weibull distributions. In other words, the influence of threshold stress becomes more serious as the Weibull modulus $m_{3 p}$ increases, and thus threshold stress should be taken into account in the fit of strength data, or some additional examinations are needed if the two-parameter Weibull distribution is used.

\section{Conclusions}

A simple quantitative procedure has been proposed in this paper, which can be implemented to highlight the effects of threshold stress on the estimation of the Weibull statistics. The results obtained from both numerical simulations and real data show that, as long as sample data are limited in number and threshold stress is not too large, the two-parameter Weibull distribution is still a preferred choice. But, as the Weibull modulus and threshold stress increase, a good compromise should be made between simplicity of the two-parameter Weibull distribution and applicability of the three-parameter Weibull distribution.

\section{Acknowledgments}

We gratefully acknowledge Dr. T. Lube for supplying the strength data of ceramics. One of the authors (C. Lu) would like to thank Professor D. Vere-Jones for many valuable discussions.

Table II. Fitted Parameters and Calculated AIC Values by Different Distributions

\begin{tabular}{|c|c|c|c|c|c|c|c|c|c|}
\hline Specimen & $N$ & $m_{3 \mathrm{p}}$ & $\sigma_{03 \mathrm{p}}$ & $\sigma_{\text {th }}$ & $(\mathrm{AIC})_{3 \mathrm{p}}$ & $m_{2 \mathrm{p}}$ & $\sigma_{02 \mathrm{p}}$ & $(\mathrm{AIC})_{2 \mathrm{p}}$ & $\Delta$ (AIC) \\
\hline Soda-lime & 24 & 5.01 & 105.70 & 23.25 & 221.96 & 5.74 & 128.70 & 220.86 & -1.10 \\
\hline $\mathrm{Si}_{3} \mathrm{~N}_{4}$ & 55 & 13.75 & 924.30 & 9.24 & 637.77 & 13.89 & 933.56 & 635.78 & -1.99 \\
\hline $\mathrm{SiC}$ & 75 & 8.01 & 310.70 & 65.25 & 779.83 & 9.62 & 376.20 & 778.31 & -1.52 \\
\hline
\end{tabular}




\section{References}

${ }^{1}$ B. R. Lawn, Fracture of Brittle Solids, 2nd Ed. Cambridge University Press, Cambridge, U.K., 1993.

${ }^{2}$ J. B. Wachtman, Mechanical Properties of Ceramics. Wiley, New York, 1996.

${ }^{3}$ S. M. Weiderhorn and E. R. Fuller Jr., "Structural Reliability of Ceramic Materials," Mater. Sci. Eng., 71, 169-86 (1985).

${ }^{4}$ W. Weibull, "A Statistical Theory of the Strength of Materials," Proc. R. Swed. Inst. Eng. Res., 151, 1-45 (1939).

${ }^{5}$ W. Weibull, "A Statistical Distribution Function of Wide Applicability," J. Appl. Mech., 18, 293-97 (1951).

${ }^{6}$ V. J. Tennery, K. Breder, M. K. Ferber, and M. G. Jenkins, "Tensile Fracture Behavior of Two Types of Silicon Nitride Specimen Geometries Conducted by Ten U.S. Groups," J. Am. Ceram. Soc., 83, 1186-91 (2000).

${ }^{7}$ S. L. Fok, B. C. Mitchell, J. Smart, and B. J. Marsden, "A Numerical Study on the Application of the Weibull Theory to Brittle Materials," Eng. Fract. Mech., 68 1171-79 (2001).

${ }^{8} \mathrm{H}$. Peterlik, "Comment on 'Relationship between the Estimated Weibull Modulus and the Coefficient of Variation of the Measured Strength for Ceramics'," J. Am Ceram. Soc., 83, 1013-14 (2000).

${ }^{9}$ H. Akaike, "On Entropy Maximization Principle"; pp. 27-41 in Applications of Statistics. Edited by P. R. Krishnaiah. North-Holland, Amsterdam, Netherlands, 1977.

${ }^{10}$ Y. Sakamoto, M. Ishiguro, and G. Kitagawa, Akaike Information Criterion Statistics. Reidel, Dordrecht, Netherlands, 1983.

${ }^{11}$ C. Lu, D. Vere-Jones, and H. Takayasu, "Avalanche Behavior and Statistical Properties in a Microcrack Coalescence Process," Phys. Rev. Lett., 82, 347-50 (1999).
${ }^{12} \mathrm{C}$. Lu and D. Vere-Jones, "Statistical Analysis of Synthetic Earthquake Catalogs Generated by Models with Various Levels of Fault Zone Disorder," J. Geophys. Res., 106, 11115-25 (2001).

${ }^{13}$ D. A. Reed and E. R. Fuller Jr., "Glass Strength Degradation Under Fluctuating Loads," J. Struct. Eng., 111, 1460-67 (1984).

${ }^{14}$ J. E. Ritter, P. B. Oates, E. R. Fuller Jr., and S. M. Wiederhorn, "Proof Testing of Ceramics, Part 1, Experiment," J. Mater. Sci., 15, 2275-81 (1980).

${ }^{15}$ E. R. Fuller Jr., S. M. Wiederhorn, J. E. Ritter, and P. B. Oates, "Proof Testing of Ceramics, Part 2, Theory," J. Mater. Sci., 15, 2282-95 (1980).

${ }^{16} \mathrm{C}$. Lu and H. Xie, "A Physical Limit of Weibull Modulus in Rock Fracture," Int. J. Fract., 72, R55-58 (1995).

${ }^{17}$ Y. Bai, Y. Wei, M. Xia, and F. Ke, "Weibull Modulus for Diverse Strength Due to Sample Specificity," Theor. Appl. Fract. Mech., 34, 211-16 (2000).

${ }^{18} \mathrm{P}$. D. Warren, "Fracture of Brittle Materials: Effects of Test Method and Threshold Stress on the Weibull Modulus," J. Eur. Ceram. Soc., 21, 335-42 (2001).

${ }^{19}$ R. Danzer, T. Lube, and P. Supancic, "Monte Carlo Simulations of Strength Distributions of Brittle Materials-Type of Distribution, Specimen and Sample Size," Z. Metallkd., 92, 773-83 (2001)

${ }^{20}$ R. H. Doremus, "Fracture Statistics: A Comparison of the Normal, Weibull, and Type I Extreme Value Distributions," J. Appl. Phys., 54, 193-98 (1983).

${ }^{21}$ R. Danzer and T. Lube, "Fracture Statistics of Brittle Materials: It Does Not Always Have to be Weibull Statistics"; pp. 683-88 in Ceramic Materials and Components for Engines. Edited by K. Niihara. Japan Fine Ceramics Association, Tokyo, Japan, 1998.

${ }^{22}$ R. Danzer, "A General Strength Distribution Function for Brittle Materials," J. Eur. Ceram. Soc., 10, 461-72 (1992). 